\title{
Reflets
}

Revue ontaroise d'intervention sociale et communautaire

\section{Pénurie infirmière : mythe ou réalité?}

\section{Danielle Dorschner}

Volume 5, numéro 2, automne 1999

La santé des francophones de l’Ontario

URI : https://id.erudit.org/iderudit/026281ar

DOI : https://doi.org/10.7202/026281ar

Aller au sommaire du numéro

Éditeur(s)

Reflets : Revue ontaroise d'intervention sociale et communautaire

ISSN

1203-4576 (imprimé)

1712-8498 (numérique)

Découvrir la revue

Citer cet article

Dorschner, D. (1999). Pénurie infirmière : mythe ou réalité? Reflets, 5(2),

234-238. https://doi.org/10.7202/026281ar

Tous droits réservés (C) Reflets : Revue ontaroise d'intervention sociale et communautaire, 1999

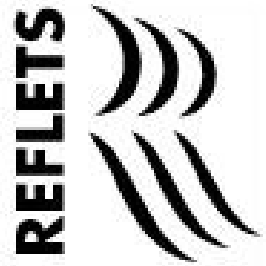

Ce document est protégé par la loi sur le droit d'auteur. L'utilisation des services d'Érudit (y compris la reproduction) est assujettie à sa politique d'utilisation que vous pouvez consulter en ligne.

https://apropos.erudit.org/fr/usagers/politique-dutilisation/ 


\section{Pénurie infirmière : mythe ou réalité?}

\section{D anielle $D$ orschner}

La pénurie d'infirmières n'est pas chose nouvelle pour notre profession. À l'heure actuelle, cette pénurie est un syndrome qui peut être relié à plusieurs facteurs, les uns plus complexes que les autres. $C$ et article tentera d'examiner ces facteurs ainsi que les opportunités qui s'offrent aux infirmières à l'aube de ce nouveau millénaire. De plus, nous examinerons les solutions possibles pour contrer cette pénurie et revaloriser cette profession qui noustient tellement à coeur.

Lors de la conférenceA cadie-Sherbrooke, en juin 1999, Ginette R odger-Lemire a dressé un portrait de l'infirmière d'aujourd'hui . À I'heure actuelle, il y a plus de 260000 infirmières autorisées au C anada, ce qui représente près de $75 \%$ de tous les professionnels de la santé. La majorité de ces infirmières (62 \%) travaillent en milieu hospitalier. Près de $12 \%$ œuvrent au sein de centres de soins de longue durée, $7 \%$ en santé communautaire et $6 \%$ en soins à domicile. Les femmes dominent cette profession, car plus de $95 \%$ des effectifs sont des femmes. En ce qui a trait à la formation et l'éducation de base des infirmières, $77 \%$ de cellesci ont obtenu un diplôme de niveau collégial. De plus, seulement la moitié détiennent un emploi à temps plein, ce qui veut dire que la moitié des infirmières travaillent à temps partiel ou sur appel. De 1994 à 1998, le R N AO estime à 8 000, la perte de postes d'infirmières en 0 ntario. Ces postes ont été éliminés suite aux coupures budgétaires et aux restructurations dans les hôpitaux.

À quoi ressemblera cette pénurie dans les années à venir? L'A ssociation des infirmièreset infirmiers du C anada (AIIC) prédit 
que la demande pour des services infirmiers augmentera de $46 \%$ durant la prochaine décennie. En 2011, la pénurie de personnel infirmier pourrait atteindre 113000 personnes. L'AIIC attribue cette pénurie appréhendée à la moyenne d'âge élevé chez les infirmières, soit 45 ans, à la population vieillissante, ce qui augmentera la demande en soins infirmiers et à une diminution des nouvelles diplômées, ce qui ne favorise pas la création d'une relève. Durant les dernières années, le nombre de nouvelles graduées est passé de 10000 à 5000 par année. De plus, les infirmières ressentent des manques au niveau professionnel et souffrent d'épuisement, ce qui les incitent à quitter la profession. Le recrutement de nouvelles infirmières n'est pas facile.

Q uelle est la cause de ces épuisements professionnels? Les infirmières affirment vivre un niveau élevé de stress et de changements danslesmilieux de travail.Au cours des dix dernières années, les compressions budgétaires ont beaucoup affecté notre profession. Le nombre de patients à charge est de plus en plus élevé et les patients sont de plus en plus gravement malades.

Pour réduire les coûts, certaines institutions et agences ont embauché des «multi-techniciennes » et des travailleurs non licenciés. Le rôle des infirmières a subi de nombreuses transformations au cours des ans, sans pour autant que les infirmières intégrées au système de santé aient reçu de formation additionnelle ou de suivi professionnel pour s'acquitter de ces nouveaux rôles. La technologie change tellement vite qu'il est difficile de se tenir à jour. Les infirmières sont aussi préoccupées par la qualité des soins et la sécurité des patients.

Tout n'est pas noir dans le monde du nursing. En effet, on note que le baccalauréat universitaire est davantage exigé comme critère d'admission à l'exercice de la profession, ce qui est en soi remarquable. Les programmes de maîtrise et de doctorat sont de plus en plus populaires auprès des infirmières qui désirent approfondir leurs connaissances en nursing. En O ntario, àTerre$\mathrm{N}$ euve et en A lberta, les infirmières praticiennes sont maintenant reconnues comme un rouage important des soins de santé primaire. 
Durant les prochaines années, 25 millions de dollars seront consacrés au recrutement de nouvelles infirmières, à rehausser l'image de la profession et à la recherche en sciences infirmières. En 1998, le ministre fédéral de la santé, l'H onorable Allan R ock, a annoncé la nomination de Dr. Judith Shamian au poste de directrice exécutive de la Politique des soins infirmiers de Santé Canada.

Q uelles sont les solutions possibles à ce scénario de pénurie de personnel infirmier? Q ue peuvent faire les instances gouvernementales, nos associations et les infirmières pour prévenir une crise au niveau de notre profession?

\section{Les instances gouvernementales}

Les promesses des politiciens et des bureaucrates d'embaucher des infirmières n'est pas l'unique solution. Le M inistère de la santé de l'O ntario a récemment promis d'embaucher 10000 infirmières pour combler le déficit de personnel infirmier. M ais la question demeure: où irons-nous chercher toutes ces infirmières? Le nombre de nouvelles diplômées n'est déjà pas suffisant pour répondre à cette demande. Et il sera de plus en plus difficile d'attirer vers la profession un nombre de personnes qui est désillusionné sur le sort réservé aux infirmières et fatigué par la vague de changements, de compressions et de restructurations dans la système de santé.

II faut plutôt revaloriser la profession d'infirmières auprès des jeunes qui désirent faire carrière dans le domaine des soins de la santé. Le gouvernement se doit d'avoir une vision et un plan d'action stratégique pour répondre aux besoins des infirmières. Le rapport du N ursing Task Force a été bien reçu par le gouvernement ontarien et son ministre de la santé. Le N ursing Task Force a interrogé de nombreux individus, des associations et des regroupements d'infirmières afin de connaître leurs préoccupations, leurs opinions et leurs suggestions pour pallier ce problème de recrutement et de morosité ambiante. Les 
infirmières ont exprimé de vive voix leurs frustrations et ont proposé des solutions rafraîchissantes et créatives. II suffit maintenant d'agir sur la base des nombreuses recommandations faites par les membres du N ursing Task Force.

Apprécier l'infirmière à sa juste valeur peut avoir un aspect bénéfique, non seulement auprès des jeunes, mais aussi auprès de celles qui sont déjà en poste et qui trouveraient là un souffle nouveau pour continuer à exercer leur profession. II ne s'agit pas seulement de rémunération adéquate. II faut reconnaitre le rôle unique et important de l'infirmière. II faut les impliquer dans les processus décisionnels. La formation continue, la recherche infirmière et le respect de notre discipline à l'intérieur de nos milieux de travail doivent être valorisés. De plus, afin de retenir les nouvelles diplômées dans les centres hospitaliers, on devrait établir des programmes de parrainage et des rôles de mentors pour guider les premiers pas de ces nouvelles recrues.

\section{Nos associations professionnelles}

N os représentantes professionnelles peuvent prendre part aux solutions pour résoudre cette crise. D urant les dix dernières années, nos associations professionnelles, parlant d'une voix forte, ont été plus actives au niveau politique. $N$ ous avons profité de l'occasion pour faire valoir nos idées et nos préoccupations. $\mathrm{N}$ ous avons démontré que nous sommes des leaders créatifs et des grands défenseurs de nos clients.

C ependant, il reste encore du travail à faire. Les associations et les groupes qui représentent les infirmières ont l'obligation de participer aux efforts de recrutement. Le R egroupement des intervenantes et intervenants francophones en santé et en services sociaux de l'O ntario (R IFSSSO ) s'est engagé dans cette voie. En développant le programme J'me lance en santé, ce regroupement entend favoriser le recrutement d'intervenants francophones en santé. Le R IFSSSO, avec l'appui financier du ministère de la Santé de l'O ntario, a publié des dépliants décrivant plusieurs possibilités 
de carrières dans le domaine de la santé et des services sociaux et ont récemment créé un site web. Les organismes scolaires et professionnels peuvent utiliser ces outils afin de recruter nosjeunes du secondaire. L'A ssociation desinfirmières et infirmiersautorisés de I'O ntario (AIIAO) a développé un programme similaire dans les écoles secondaires de la province, en visant plus particulièrement les orienteurs scolaires.

II faut aussi continuer nos efforts de lobby et saisir chaque opportunité de faire valoir nos préoccupations. Ça ne suffit pas d'informer les gouvernements, il faut aussi informer le public. $\mathrm{N}$ ous avons un devoir de partager avec la collectivité les enjeux et nos inquiétudes face aux transformations de notre système de santé. II faut aussi peindre une image positive de la profession infirmière qui fait appel au professionnalisme, à la compétence et à l'intelligence de la personne.

Vu l'importante pénurie qui s'annonce, le rôle de l'infirmière dans notre système de santé doit être repensé. N ous avons trop longtemps dénoncé l'embauche de para-professionnels de la santé qui se substituaient aux infirmières. II serait peut-être temps d'examiner la contribution de ces derniers et leurs relations avec les infirmières? Ainsi, l'infirmière pourrait jouer un rôle de coordinatrice des soins, de gestionnaire et de leader d'équipe de soins. C e rôle élargi de l'infirmière réduirait d'autant leur nombre pour combler la pénurie appréhendée. De plus, avec un nombre croissant d'infirmières bachelières ou détentrices de maîtrise et de doctorats, la profession est appelée à se spécialiser davantage, à se développer et à grandir.

La tâche de trouver une solution à la pénurie d'infirmières ne repose pas seulement sur les gouvernements. $N$ ous devons agir maintenant au sein de nos organisations professionnelles et de nos communautés. Ensemble, les infirmières forment une masse importante dans le domaine des soins de santé. C 'est en continuant de défendre nos droits que la profession et la collectivité en bénéficieront. 